\title{
Universiteit
}

Leiden

The Netherlands

\section{Virtual reality as a research method in criminology}

Cornet, L.J.M.; Gelder, J.L. van; Barnes, J.C.; Forde, D.R.

\section{Citation}

Cornet, L. J. M., \& Gelder, J. L. van. (2021). Virtual reality as a research method in criminology. In J. C. Barnes \& D. R. Forde (Eds.), The encyclopedia of research methods in criminology and criminal justice (pp. 893-900). Hoboken: Wiley-Blackwell.

doi:10.1002/9781119111931.ch174

Version: Publisher's Version

License: $\quad$ Licensed under Article 25fa Copyright Act/Law (Amendment Taverne)

Downloaded from: https://hdl.handle.net/1887/3264300

Note: To cite this publication please use the final published version (if applicable). 


\title{
Virtual Reality as a Research Method in Criminology
}

\author{
Liza Cornet ${ }^{1}$ and Jean-Louis van Gelder ${ }^{1,2}$ \\ ${ }^{1}$ Institute of Education and Child Studies, Faculty of Social and Behavioural Sciences, Leiden University, \\ Leiden, The Netherlands \\ ${ }^{2}$ Max Planck Institute for the Study of Crime, Security and Law, Freiburg, Germany
}

This work was supported by a European Research Council Consolidator Grant (Grant Number 772911- CRIMETIME).

\section{Introduction}

Staple research methods in criminology, such as interviews, surveys, and official registration data, tend to inquire about past events and/or rely on introspection and self-disclosure. Hence such methods tend to measure criminal behavior indirectly and are proxies of actual behavior at best. Observational methods remedy these limitations to some extent, but when it comes to the study of criminal conduct, such methods pose additional challenges for ethical and practical reasons. Evidently, few Institutional Review Boards (IRBs) would approve research projects that intend to observe crime as it takes place, and by the same token few criminals are likely to welcome researchers to watch them on the job. Furthermore, observational studies are limited in the extent to which they allow for experimental variation. Experiments such as those undertaken in university labs, in contrast, are restricted in terms of the type and severity of the behavior studied, and the sterile and often contrived nature of many lab experiments restricts their ecological validity. The methodological toolbox of the criminologist, in short, still lacks a multitool properly equipped to fill these gaps and to examine behavior as it takes place.
A relatively novel technology, virtual reality (VR), that has seen dramatic development in recent years, actually allows researchers to approximate the criminal event and study criminal behavior in real time. The merit of VR technology for criminological research, and for social science research more broadly, resides in the technology's capacity to create "systematic human testing, training, teaching, and treatment environments that allow for the precise control of complex, multi-sensory, dynamic 3D stimulus presentations" (Rizzo and Koenig 2017, p. 4). Within such interactive virtual environments, user activity can be tracked, recorded, and analyzed in great detail and as it occurs. Furthermore, VR can be used in combination with other technologies, such as eye-tracking, physiological measurements, including heart rate and galvanic skin response, and other methods of data collection, e.g. by having users in the virtual environment think out loud while they perform an action (see Meenaghan et al. 2018), to provide more encompassing views of offender behavior and its key drivers.

Although still in its infancy as a research method in criminology, there is an established research tradition using VR in (mental) healthcare and other social sciences, and the technology has also been used to study a 
variety of phenomena relevant to criminologists, such as stereotyping and racial bias (Dotsch and Wigboldus 2008), disorderly conduct (Toet and van Schaik 2012), obedience and authoritarianism (Slater et al. 2006), aggression (Slater et al. 2013; van Gelder et al. 2019), moral judgment (Navarrete et al. 2012), victimization risk (Park et al. 2012), and recently also delinquency (Van Gelder et al. 2013) and crime (Nee et al. 2019).

In this entry, we give an overview of VR technology and its potential as a research method for application in criminological research. Below, we will first provide a brief explanation of the term "virtual reality" and its core features along with some of the possibilities it offers for social science research, and for crime research in particular. Subsequently, we will review published work that has employed VR as a research method to study criminal decision-making, fear of victimization, and the bystander effect. We will conclude with a short discussion of practical and ethical considerations involved in the use of VR in crime research, gaps in the current knowledge base, and identifying relevant areas for future research.

\section{Virtual Reality: What Is It?}

The term "virtual reality" was coined in the late 1980s and refers to an artificial, often computer-generated, three-dimensional environment that replaces the physical environment (Fox et al. 2009). ${ }^{1}$ Virtual environments can be experienced via different types of digital media, such as desktop computers, laptops, tablets, and smartphones. As Rizzo

\footnotetext{
1 A relevant distinction is that between animated, or rendered, $\mathrm{VR}$ and $360^{\circ}$ video, which is a branch of VR that uses multiple cameras to record the full $360^{\circ} \times 180^{\circ}$ field of view to create an immersive experience. Although experienced through a head-mounted display, there is discussion as to whether it should be seen as VR as it allows for limited interaction only (see Slater and Sanchez-Vives 2016).
}

and Koenig (2017, p. 2) observe: "An engaged VR user experience can be accomplished using combinations of a wide variety of interaction devices, sensory display systems, and content presented in the virtual environment." So-called immersive VR, which is the focus of this entry, is generally experienced via a head-mounted display (HMD) rather than a flat screen, that shuts the user off from real-world visual input. The HMD in combination with a rendering computer allows for tracking the exact position of the user and feeding it back to her in a naturalistic way. Additional body trackers can ensure the correspondence between user movements in the real world and the simulated world.

The term "immersion" denotes the objective amount and quality of the perceptual input provided to participants through hardware (Bombari et al. 2015; Slater 2003). As Slater $(2003$, p. 1) argues: "The more that a system delivers displays (in all sensory modalities) and tracking that preserves fidelity in relation to their equivalent realworld sensory modalities, the more that it is immersive." Whereas most commonly, immersion is restricted to the visual and auditory input via the HMD, additional layers can be added, for example through haptic gloves, kinetic suits, and incorporated scents.

The psychological corollary of immersion is known as "presence," which refers to the subjective sense of being in the virtual environment rather than the physical environment where one's body is located (Slater 2004; Slater et al. 2006). Since the human mind cannot be present in different places at the same time, feeling present in the virtual world implies psychological absence in the real world (Bailenson 2018).

\section{Virtual Reality in Criminological Research}

Different studies have summarized the advantages that VR offers for social (neuro) 
science (see Pan and Hamilton 2018; Parsons 2015), which bears relevance for criminological research as well. One of the most important advantages is that the technology provides the opportunity to create ecologically valid situations to study real-time behavioral responses of (a group of) individuals. As mentioned in the introduction to this entry, in social science research, behavioral responses are frequently measured with self-report questionnaires or experimental set-ups (with actors) are created to evoke and observe subjects' reactions. However, self-report measures of behavior are subject to error and bias, because people do not always (correctly) report on things they have done or intend to do (e.g. Baumeister et al. 2007). Furthermore, as described, there are practical and ethical limitations in the extent to which experimental scenarios can mirror real-life situations (e.g. recruiting actors and creating the right environmental circumstances can be challenging). With VR, all kinds of situations can be created that are difficult to realize, expensive, or simply impossible to create in real life (Cornet and Van Gelder 2020; Van Gelder et al. 2014). There is convincing evidence that it may take only a few minutes before users react to the virtual world as if it were real (Pan and Hamilton 2018, p. 2; Slater 2009; Slater and Sanchez-Vives 2016).

Besides its ability to create situations with mundane realism, and hence establish high levels of ecological validity, VR enables the manipulation of specific aspects of the virtual environment, thereby providing high levels of experimental control (Pan and Hamilton 2018, p. 2). Take, for example, a situation in which a researcher is interested in how the gender and ethnic background of a victim affects people's intervention behavior during a violent incident. This requires either high levels of participants' imagination or multiple actors of different gender/ethnic backgrounds (Pan and Hamilton 2018). In VR, gender and skin color of virtual representations of people (i.e. avatars) can be changed in a relatively straightforward manner, keeping all other factors constant. Hence, it is possible to create infinite combinations of variables and test them against each other (Pan and Hamilton 2018, p. 2). Furthermore, once created, a VR scenario can be shared and used repeatedly to allow testing of a large number of participants across different research laboratories or even different countries. This allows for direct replication and thus can contribute to the reproducibility of studies (Pan and Hamilton 2018, p. 3).

In spite of its potential, criminological research using VR is at a very early stage. Yet, as mentioned in the introduction, the technology has been extensively used in other social science domains and for mental health, to study phenomena that have relevance for crime researchers, such as aggression and moral judgment. Below, we identify and discuss three areas with relevance for criminologists: criminal decision-making, bystander intervention, and fear of crime and victimization.

\section{Unhiding the Hidden: Using VR to Study Criminal Decision-making}

As explained at the outset of this entry, VR offers the possibility to mimic the real world and to subsequently observe behavior in real time. Given that criminal conduct almost per definition takes place out of sight, the ability of VR to simulate the real world offers novel possibilities to study criminal decision-making.

Traditionally, the study of criminal choice has been carried out using vignette or scenario research. The scenario method is a versatile, effective, low-cost, and efficient method of data collection about how people would act in situations that are outside of the purview of other methods, for example because of their sensitive nature, prohibitive cost, or infrequent occurrence (Collett and Childs 2011). Yet vignettes rely on the ability 
of research participants to imagine themselves in a described situation, whereas VR can actually immerse them in it. In a recent study, Van Gelder et al. (2019) proposed and tested a "virtual reality scenario method." The authors developed a 360-degree video version of a "bar fight" scenario (Mazerolle et al. 2003; Schoepfer and Piquero 2006) and tested it against the commonly used written version of that same scenario. They found that participants in the VR condition experienced higher levels of presence and higher intentions to aggress, and rated the scenario as more realistic. Furthermore, both presence and anger were found to mediate the relation between condition and intentions to aggress.

In the ongoing Virtual Burglary Project (VBP), researchers from the University of Portsmouth in England, and the Vrije Universiteit Amsterdam and the University of Twente in the Netherlands use virtual environments to study the decision making of burglars. In the VBP, incarcerated burglars, and sometimes other groups, are invited to scope a residential neighborhood for opportunities, select a target to burglarize and/or to commit a burglary in VR, and to go about it as they would do in the real world (Nee et al. 2015, 2019; Van Gelder et al. 2017). The VR system tracks all their behavior in real time, such as where they look, how they walk around, which target they select, where they enter it, and how they go about doing the burglary once inside. These studies have shown that burglary behavior in the real world corresponds to a large degree with burglary behavior in the simulated environment. Furthermore, research in the VBP has shown clear differences in the decision-making of burglars compared with other groups in ways commensurate with expertise in other behavioral domains (Nee et al. 2019).

Another type of approach is provided in a study by Van Gelder et al. (2013). Rather than using VR to focus on the decision process, this study employs VR to influence sub- sequent decision-making. The authors used immersive VR to have participants meet their future self. The authors hypothesized that strengthening the vividness of the future self could reduce delinquency. In this study, the authors developed a VR environment in which participants faced a realistic digital version of either their future, age-progressed self or their contemporary self (control condition) in a virtual mirror. Participants who interacted with a realistic digital version of their future, age-progressed self in a virtual environment were significantly less likely than control participants to cheat on a subsequent task. The results of this study show that interacting with a future version of oneself could lead to a significant reduction in delinquent behavior.

\section{Studying the Bystander-Effect in (Sexual) Violent Emergencies}

The bystander effect refers to the well-known idea that people's feelings of responsibility to intervene in emergencies diffuses with the presence of other bystanders (e.g. Darley and Latané 1968; Fischer et al. 2011). Ideally, how people behave during critical situations is observed in real time, but this might be challenging, especially when the behavioral response to a violent emergence is the central research focus. In their article, Rovira et al. $(2009$, p. 1) review the potential of VR for studying the bystander effect in response to a violent incident and argue that VR provides an ecologically valid setting in which to study this issue while at the same time removing the problem of physical danger and overcoming the many ethical issues involved in the study of violence.

An interesting study in this regard was conducted by Slater et al. (2013), who used immersive VR to investigate whether supporters of Arsenal Football Club would intervene during a physical attack between two virtual humans in a bar. The central question of this study concerned the extent 
to which the participant, who was an unrelated bystander in the VR scenario, would try to intervene and whether the relationship with the victim would affect the helping behavior of the participant. It appears that participants in the in-group condition, in which the victim was also an Arsenal fan, made more attempts at physical and verbal intervention compared with participants in the out-group condition, in which the victim was not an Arsenal fan.

Another study was conducted by Jouriles et al. (2016), who investigated bystander behavior in response to sexual violent advances. The authors designed a VR procedure that involved virtual scenarios of various situations that could conceivably escalate to a female being sexually victimized, allowing participants to try to prevent it (Jouriles et al. 2016, p. 2). Interestingly, the observed bystander behavior during the virtual scenarios correlated with self-reported responsibility and intention to intervene as assessed before entering the VR scenarios. The study provides support for the procedure's validity to investigate the bystander effect in VR.

Despite these promising findings, it should be noted that these results do not necessarily prove that what participants do in real life matches what research finds in VR. However, these experiments cannot be carried out in reality, hence the results of these studies could be used in the process of constructing new theories (Slater et al. 2013, p. 11).

\section{Perceptions of Personal Safety}

Fear of crime is a significant factor in how people approach their environments. Park et al. (2012) conducted a study in which a semi-immersive virtual environment (projected on to a large screen) was used to examine context-specific assessments of victimization risk. Participants' decision-making in this study was assessed across a series of choices while walking through a virtual city. Participants were free to navigate the environment, with the only condition that every time they came across a decision point, they selected one of two available options (e.g. taking a wide or a narrow alley). In line with previous research, Park and colleagues found that female participants displayed greater sensitivity toward the risk of victimization compared with male participants, and there appeared to be no differences for participants of different ages.

Toet and Van Schaik (2012) compared the effects of signs of public disorder, such as litter, cameras, vandalism, and car burglary, on fear of crime, and explored the ecological validity of their virtual environment by examining whether responses to it resembled those in its real-world counterpart. Participants in this study walked through either the virtual or the real neighborhood, which was either in an orderly state or showed signs of disorder. Following their tour, they appraised the perceived safety and livability of the area. Both in the real and the simulated neighborhood, signs of physical disorder evoked associations with social disorder. Disorder did not inspire concern for personal safety in either environment but was associated with negative emotions more frequently in the virtual environment than in its real-world counterpart.

Cozens et al. (2003) conducted an interesting study investigating fear of crime with the help of 360-degree VR walkthrough scenarios of train stations located in Wales. In total, 47 participants were asked to virtually travel through a train station and to afterward state whether they feared for their personal safety. The most significant and commonly cited personal safety fears were in relation to railway station environments at night, and the most frequently cited suggested improvement for all the respondents was "better lighting" (Cozen et al. 2003, p. 130). The authors argue that utilizing more dynamic and interactive environmental stimuli represented by VR-walkthroughs can be analyzed in conjunction with existing objective indicators for 
crime (recorded crime rates) and therefore arguably represent a more holistic approach to understanding the problems of personal safety and fear of crime in and around real-life environments (Cozens et al. 2003, p. 130).

\section{The Future of Criminological Research}

In the final section of this entry, we will briefly discuss practical and ethical considerations involved in the use of VR in crime research, gaps in the current knowledge base, and relevant areas for future criminological research using this technology.

Despite the possibilities it offers for crime research, VR is not yet extensively used as a research method by criminologists. Different reasons may account for this. One reason relates to the practical issues associated with VR hardware, including costs, discomfort when wearing the headset for extended periods of time, and vulnerability to damage if used intensively and on-site. Other challenges are headaches, cybersickness, and eyestrain. Furthermore, generating adequate levels of "presence" can be challenging. For these and other reasons, such as crime researchers' lack of familiarity with the technology, the development of VR content often requires specialized knowledge, and as a result can involve substantial time and cost (see Cornet and Van Gelder 2020).

There are also gaps in the current knowledge base regarding the added value and effectiveness of VR technology. According to Pan and Hamilton (2018), "just as we do not always know if laboratory studies apply in the real world, similarly we must be cautious about claiming that VR studies, where participants still know they are in an 'experimental psychology context', will generalize to real-world interactions without that context" (p. 3). Indeed, although several studies have demonstrated correspondence between behavior in the virtual world (e.g. Nee et al. 2015, 2019; Slater et al. 2006) and that in the real world, more research is necessary to examine to what extent these findings hold over different situations and to establish boundary conditions.

Other relevant considerations with respect to the use of VR are related to the possible psychological and emotional side effects of specific VR experiences. There is evidence, for example, that very young children might develop false memories based on VR experiences (Segovia and Bailenson 2009). VR content may be used in a way different from that intended by a researcher (e.g. virtual risk situations for child abusers may be misused as child pornography). Fromberger et al. (2018, pp. 6-7) describe these and other ethical issues in their "ethical guidelines for research and clinical application of VR in forensic psychiatry."

In terms of ethics, it is important to note that the ability with VR to collect enormous amounts of data in a precise way also carries risks. For example, producers of VR headsets (e.g. HTC, Facebook), may collect data for commercial purposes and possibly share it with third parties, and users could be identified on the basis of their digital "fingerprint" (Madary and Metzinger 2016). This can generate conflicts with data privacy. Fromberger et al. (2018) observe that with the commercialization of VR equipment, companies mostly provide software that requires internet access in order to function and thus it is unknown what user data are accessed and collected online.

In its current form, VR can enrich the toolbox of criminological research methods, rather than substituting for existing measurement methods. Criminologists could use VR as a research method to further explore realtime criminal behavior as it occurs, observe people's response to scenarios that cannot be created in real life, and improve the ecological validity of traditional research methods (e.g. vignette research in VR). Hardware costs are likely to further reduce, VR hardware will become more user-friendly, and the 
possibilities for application are rapidly expanding. We have all reason to assume that the use of VR in crime research will only increase in the coming years, possibly establishing itself next to the other staple methods of data collection in criminology.

\section{References}

Bailenson, J. (2018). Experience on Demand. What Virtual Reality Is, How It Works, and What It Can Do. New York: Norton \& Company, Inc.

Baumeister, R.F., Vohs, K.D., and Funder, D.C. (2007). Psychology as the science of self-reports and finger movements: whatever happened to actual behavior? Perspectives on Psychological Science 2 (4): 396-403.

Bombari, D., Schmid Mast, M., Canadas, E., and Bachmann, M. (2015). Studying social interactions through immersive virtual environment technology: virtues, pitfalls, and future challenges. Frontiers in Psychology 6: 869.

Collett, J.L. and Childs, E. (2011). Minding the gap: meaning, affect, and the potential shortcomings of vignettes. Social Science Research 40 (2): 513-522.

Cornet, L.J.M. and Van Gelder, J.L. (2020). Virtual reality: a use case for criminal justice practice. Psychology, Crime \& Law, 1-17.

Cozens, P., Neale, R., Whitaker, J., and Hillier, D. (2003). Managing crime and the fear of crime at railway stations - a case study in South Wales (UK). International Journal of Transport Management 1 (3): 121-132.

Darley, J.M. and Latané, B. (1968). Bystander intervention in emergencies: diffusion of responsibility. Journal of Personality and Social Psychology 8 (4p1): 377.

Dotsch, R. and Wigboldus, D.H.J. (2008). Virtual prejudice. Journal of Experimental Social Psychology 44 (4): 1194-1198.

Fischer, P., Krueger, J.I., Greitemeyer, T. et al. (2011). The bystander-effect: a meta-analytic review on bystander intervention in dangerous and non-dangerous emergencies. Psychological Bulletin 137 (4): 517.

Fox, J., Arena, D., and Bailenson, J.N. (2009). Virtual reality: a survival guide for the social scientist. Journal of Media Psychology 21 (3): 95-113.

Fromberger, P., Jordan, K., and Müller, J.L. (2018). Virtual reality applications for diagnosis, risk assessment and therapy of child abusers. Behavioral Sciences \& the Law 36 (2): 235-244. van Gelder, J.-L., de Vries, R.E., Demetriou, A. et al. (2019). The virtual reality scenario method: moving from imagination to immersion in criminal decision-making research. Journal of Research in Crime and Delinquency: 0022427818819696.

Jouriles, E.N., Kleinsasser, A., Rosenfield, D., and McDonald, R. (2016). Measuring bystander behavior to prevent sexual violence: moving beyond self reports. Psychology of Violence 6 (1): 73.

Madary, M. and Metzinger, T.K. (2016). Real virtuality: a code of ethical conduct. Recommendations for good scientific practice and the consumers of vr-technology. Frontiers in Robotics and AI 3: 3.

Mazerolle, P., Piquero, A.R., and Capowich, G.E. (2003). Examining the links between strain, situational and dispositional anger, and crime: further specifying and testing general strain theory. Youth \& Society 35 (2): 131-157.

Meenaghan, A., Nee, C., Van Gelder, J.-L. et al. (2018). Getting closer to the action: using the virtual enactment method to understand burglary. Deviant Behavior 39 (4): 437-460.

Navarrete, C.D., McDonald, M.M., Mott, M.L., and Asher, B. (2012). Virtual morality: emotion and action in a simulated three-dimensional "trolley problem". Emotion 12 (2): 364.

Nee, C., White, M., Woolford, K. et al. (2015). New methods for examining expertise in burglars in natural and simulated environments: preliminary findings. Psychology, Crime \& Law 21 (5): 507-513.

Nee, C., van Gelder, J., Otte, M. et al. (2019). Learning on the job: studying expertise in residential burglars using virtual environments. Criminology 57 (3): 481-511.

Pan, X. and Hamilton, A.F.d.C. (2018). Why and how to use virtual reality to study human social interaction: the challenges of exploring a new research landscape. British Journal of Psychology 109 (3): 395-417.

Park, A., Clare, J., Spicer, V. et al. (2012). Examining context-specific perceptions of risk: 
exploring the utility of "human-in-the-loop" simulation models for criminology. Journal of Experimental Criminology 8 (1): 29-47.

Parsons, T.D. (2015). Virtual reality for enhanced ecological validity and experimental control in the clinical, affective and social neurosciences. Frontiers in Human Neuroscience 9 (660) https://doi.org/10.3389/fnhum.2015.00660.

Rizzo, A. and Koenig, S.T. (2017). Is clinical virtual reality ready for primetime? Neuropsychology 31 (8): 877.

Rovira, A., Swapp, D., Spanlang, B., and Slater, M. (2009). The use of virtual reality in the study of people's responses to violent incidents. Frontiers in Behavioral Neuroscience 3: 59.

Schoepfer, A. and Piquero, A.R. (2006). Self-control, moral beliefs, and criminal activity. Deviant Behavior 27 (1): 51-71.

Segovia, K.Y. and Bailenson, J.N. (2009). Virtually true: children's acquisition of false memories in virtual reality. Media Psychology 12 (4): 371-393.

Slater, M. (2003). A note on presence terminology. Presence Connect 3 (3): 1-5.

Slater, M. (2004). How colorful was your day? Why questionnaires cannot assess presence in virtual environments. Presence Teleoperators and Virtual Environments 13 (4): 484-493.

Slater, M. (2009). Place illusion and plausibility can lead to realistic behaviour in immersive virtual environments. Philosophical Transactions of the Royal Society, B: Biological Sciences 364 (1535): 3549-3557.

Slater, M. and Sanchez-Vives, M.V. (2016). Enhancing our lives with immersive virtual reality. Frontiers in Robotics and AI 3: 74.

Slater, M., Antley, A., Davison, A. et al. (2006). A virtual reprise of the Stanley Milgram obedience experiments. PLoS One 1 (1): e39.

Slater, M., Rovira, A., Southern, R. et al. (2013). Bystander responses to a violent incident in an immersive virtual environment. PLoS One 8 (1): e52766.

Toet, A. and van Schaik, M.G. (2012). Effects of signals of disorder on fear of crime in real and virtual environments. Journal of Environmental Psychology 32 (3): 260-276.

Van Gelder, J.-L., Hershfield, H.E., and Nordgren, L.F. (2013). Vividness of the future self predicts delinquency. Psychological Science 24 (6): 974-980.

Van Gelder, J.-L., Otte, M., and Luciano, E.C. (2014). Using virtual reality in criminological research. Crime Science 3 (1): 10.

Van Gelder, J.-L., Nee, C., Otte, M. et al. (2017). Virtual burglary: exploring the potential of virtual reality to study burglary in action. Journal of Research in Crime and Delinquency 54 (1): 29-62. 